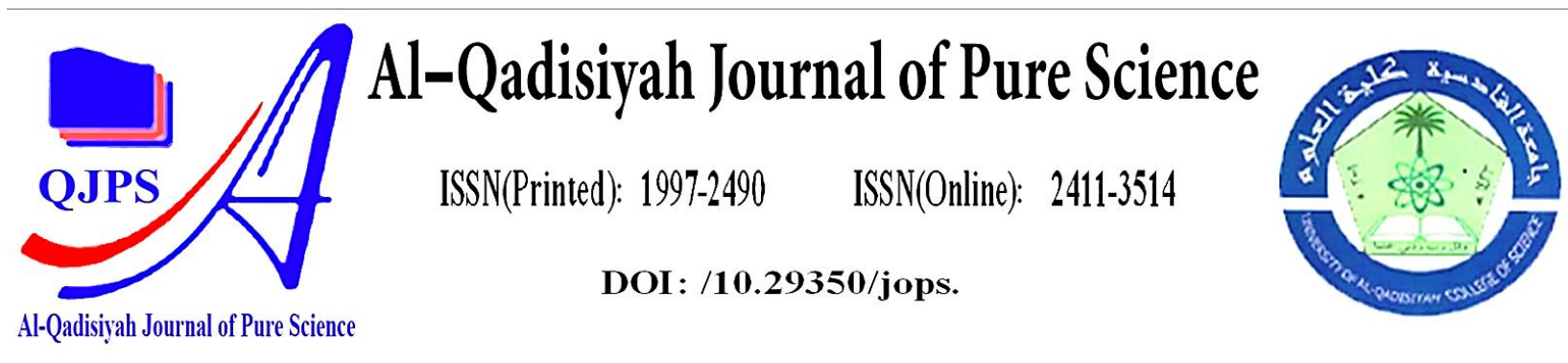

http://qu.edu.iq/iournalsc/index.php/IOPS

\title{
Quantitative and Qualitative Comparison Study between Classification Methods on normal and abnormal (with a coronavirus ) lung images
}

\begin{tabular}{|c|c|}
\hline Authors Names & ABSTRACT \\
\hline $\begin{array}{l}\text { a.Hala Kadhim hasan } \\
\text { b.Ayad A.AL-Ani } \\
\text { c.Noor Z. AlKhazraji } \\
\text { Article History } \\
\text { Received on: } 11 / 8 / 2021 \\
\text { Revised on: } 28 / 8 / 2021 \\
\text { Accepted on: } 30 \text { /8 / } 2021 \\
\text { Keywords: } \\
\text { skewness, Covid-19. unSupervisors } \\
\text { classification, Supervisors } \\
\text { classification, } \\
\text { entropy, correlation } \\
\text { DOI: https://doi.org/10.29350/ } \\
\text { jops.2021.26. } 3.1438\end{array}$ & $\begin{array}{l}\text { Classification worries with establishing criteria that may be wont to establish } \\
\text {,distinguish completely different populations of objects that seem in pictures. In this } \\
\text { paper Supervised and unsupervised classification applied on normal, abnormal (with } \\
\text { an coronavirus) a ct- lung images (took from Al shaikh zaeid Hospital) to study the } \\
\text { quantitative and qualitative properties of these two categories. The analysis of } \\
\text { performance with default quantitative parameters revealed that (kurtosis, skewness, } \\
\text { entropy, Stander deviation (STD), mean). We found that:qualitative (from an } \\
\text { appearance of the image) of abnormal lung images after applying Supervisors } \\
\text { classification are better than qualitative of abnormal lung images after applying } \\
\text { unsupervisors classification to detect the virus with a white color in the lower lobes of } \\
\text { the lung. From quantitative Properties such as (kurtosis, skewness) of original lung } \\
\text { images are similar in rising to resulted value after applying Supervisors classification } \\
\text { on it, so Supervisors method is better than unSupervisors method to distinguishing } \\
\text { between normal and abnormal lung images. }\end{array}$ \\
\hline
\end{tabular}

\section{Introduction}

Classification is essentially a complementary function that lies at the "high end" (i.e., that require the most complicated algorithms) in the field of image analysis. Generally, computers tend to be better than humans at classification because they are not distracted by random variations in noncritical parameters and can extract meaningful statistical behavior to isolate groups. There are two basic approaches to classification, supervised, and non-supervised depending on whether or not a set of prototypes is available[1]: 


\section{Supervised Learning}

Supervised learning (or supervised classification) is distribution-free or applied mathematics. Distribution-free ways don't need information of any priori probability distribution functions and are supported reasoning and heuristics. applied mathematics techniques are supported probability distribution models, which can be parametric (such as Gaussian distributions) or nonparametric [1]:

Distribution-free classification. Suppose there are K different objects or pattern categories $\mathrm{Si}, \mathrm{S} 2, \ldots \cdot \mathrm{Sb} \ldots, S_{k}$. Every categories is characterized by $M_{k}$ prototypes, that have( $\mathrm{N} \mathrm{x}$ 1) feature vectors $y_{m}^{(k)}, \mathrm{m}=1, \ldots, M_{k}$. Let x denote an $\mathrm{N}$ x 1 feature vector obtained from the observed image. A fundamental function in pattern recognition is termed the discriminant function. It is defined such that the kth discriminant function $g_{k}(\mathrm{x})$ takes the maximum value if $\mathrm{x}$ belongs to category $\mathrm{k}$, that is, the decision rule is

$$
\mathrm{g}_{\mathrm{k}}(\mathrm{x})>\mathrm{g}_{\mathrm{x}}(\mathrm{x}) \quad \mathrm{k} \neq \mathrm{i} \leftrightarrow \mathrm{x} \in \mathrm{gS}_{\mathrm{k}}
$$

For a $\mathrm{K}$ category problem, we need $\mathrm{K}-1$ discriminant functions. These functions divide the $\mathrm{N}$-dimensional feature space into $\mathrm{K}$ completely different regions with a maximum of $\mathrm{K}$ ( $\mathrm{K}$ 1)/2 hypersurfaces. The partitions become hyperplanes if the discriminant function is linear, that is, if it's the shape:

$$
g_{k}(x)=a_{k}^{T} x+b_{k}
$$

Such a function arises, for example, when $\mathrm{x}$ is classed to the category whose centroid is nearest in Euclidean distance to it . The associated classifier is termed the minimum mean (Euclidean) distance classifier.

\section{Nonsupervised Learning}

In nonsupervised learning, we have a tendency to decide to determine clusters or natural groupings in the feature space. A cluster could be a set of points within the feature space for that their local density is large (relative maximum) compared to the density of feature points within the surrounding region. Clustering techniques are helpful for image segmentation and for the classification of raw data to establish categories and prototypes. Clustering is also a helpful vector quantization technique for the compression of images [1].

Similarity measure approach. The success of cluster techniques rests on the partitioning of the feature area into cluster subsets. A general cluster rule is predicated on split and merge concepts. Using a similarity measure, the input vectors area unit divided into subsets. Each partition is tested to examine whether or not or not the subsets area unit sufficiently distinct. Subsets that do not appear to be sufficiently distinct area unit united. The procedure is perennial on each of the subsets till no a lot of subdivisions result or another convergence criterion is satisfied. Thus, a similarity measure, a distinctiveness take a look at, and a stopping rule area unit required to outline a cluster rule. For any two feature vectors $x_{i}$ and $x_{j}$, some of the commonly used similarity measures are [1]:

$$
\begin{gathered}
\text { dot product: }\left\langle\mathrm{x}_{\mathrm{i}}, \mathrm{x}_{\mathrm{j}}\right\rangle=\mathrm{x}_{\mathrm{i}}^{\mathrm{T}} \mathrm{x}_{\mathrm{j}} \\
\text { similarity rule: } \mathrm{S}\left(\mathrm{x}_{\mathrm{i}}, \mathrm{x}_{\mathrm{j}}\right)=\frac{\left\langle\mathrm{x}_{\mathrm{i}}, \mathrm{x}_{\mathrm{j}}\right\rangle}{\left\langle\mathrm{x}_{\mathrm{i}}, \mathrm{x}_{\mathrm{j}}\right\rangle+\left\langle\mathrm{x}_{\mathrm{i}}, \mathrm{x}_{\mathrm{j}}\right\rangle-\left\langle\mathrm{x}_{\mathrm{i}}, \mathrm{x}_{\mathrm{j}}\right\rangle} \\
\text { normalized correlation: } \rho\left(\mathrm{x}_{\mathrm{i}}, \mathrm{x}_{\mathrm{j}}\right) \triangleq \frac{\left\langle\mathrm{x}_{\mathrm{i}}, \mathrm{x}_{\mathrm{j}}\right\rangle}{\sqrt{\left\langle\mathrm{x}_{\mathrm{i}}, \mathrm{x}_{\mathrm{j}}\right\rangle\left\langle\mathrm{x}_{\mathrm{j}}, \mathrm{x}_{\mathrm{i}}\right\rangle}}
\end{gathered}
$$

Several different algorithms exist for clustering based on similarity approach. Examples are given next.

An iterative method. Assume the amount of clusters, K, is known. The partitioning of the data $\mathrm{s}$ completed such that the variance of the partition is reduced. Let $\mathrm{u}_{\mathrm{k}}(\mathrm{n})$ denote the kth cluster center at the $n$th iteration and $R_{k}$ denote the region of the kth cluster at a given iteration. Initially, we have a tendency to tend to assign arbitrary values to $u_{k}(0)$. At the nth 
iteration take one amongst the data points $\mathrm{x}_{\mathrm{i}}$ and assign it to the cluster whose center is nearest thereto, that is,

$$
x_{i} \in R_{k} \leftrightarrow d\left(x_{b}, u_{k}(n)\right)=\min _{j=1 \ldots ., k}\left[d\left(x_{b}, u_{j}(n)\right]\right.
$$

where $d(x, y)$ i s the distance measure used. Recompute the cluster centers by finding the point that minimizes the distance for elements inside every cluster. Thus

$\mathrm{u}_{\mathrm{k}}(\mathrm{n}+1): \quad \sum_{\mathrm{x}_{\mathrm{i}} \in \mathrm{R}_{\mathrm{k}}} \mathrm{d}\left(\mathrm{x}_{\mathrm{i}}, \mathrm{u}_{\mathrm{k}}(\mathrm{n}+1)=\min _{\mathrm{y}} \mathrm{d}\left(\mathrm{x}_{\mathrm{i}}, \mathrm{y}\right), \mathrm{k}=1, \ldots . \mathrm{k}\right.$

The procedure is repeated for every $x_{i}$, one at a time, till the clusters and their centers remain unchanged. If $\mathrm{d}(\mathrm{x}, \mathrm{y})$ is the Euclidean distance, then a cluster center is simply the mean location of its elements. If $\mathrm{K}$ is not known, we start with a large value of $\mathrm{K}$ and so merge to $\mathrm{K}$ $-1, \mathrm{~K}-2, \ldots$ clusters by a appropriate cluster-distance [1].

Imaging.

Techniques used were computerized tomography(CT) scan for normal lung and an abnormal (with coronavirus) lung. Coronavirus disease, is an infectious disease affecting more than 2400000 in April 20, 2020 [2].

In the CT scans with coronavirus, white patches are often seen. Usually, this is often a sign of an abnormality that radiologists are call ground glass opacity of air spaces within the lungs [3]. In CT scans findings of coronavirus were ground glass opacities, crazy paving pattern, and consolidation predominantly in sub pleural locations within lower lobes [4].

In this paper we tend to adopt a Supervised and non-supervised classification method, to study the performance of some of these methods(such as An iterative method and minimum mean distance classifier method) on the normal and abnormal images by using quantitative and qualitative properties of the resulted images. Quantitative properties, tend to with numbers, statistics, formulae, and data. When we do qualitative properties, we work with descriptions [5]. quantitative Properties of Images like kurtosis, skewness, entropy, Stander deviation (STD) mean will be found in [6]

\section{Results and Discussion:}

Typical works in the literature dealing with quantitative and qualitative properties comparison between Supervised and unsupervised classification.

\section{Comparison between Supervised and unsupervised classification Qualitative properties}

Four a ct- lung images to normal (such as kafy and eeman) and abnormal (such as raad and aeman) people were used, these images have been taken from AL-shaikh Zaid Hospital.

Supervisors classification (such as minimum mean distance classifier method) are applied on normal and abnormal lung images as shown in Fig. 1

\begin{tabular}{|c|c|c|c|c|}
\hline \multicolumn{3}{|c|}{ Normal lung images } & \multicolumn{2}{|c|}{ Abnormal lung images } \\
\hline $\begin{array}{r}\text { Name } \\
\text { of } \\
\text { patiant }\end{array}$ & eeman & kafy & aeman & Raad \\
\hline
\end{tabular}




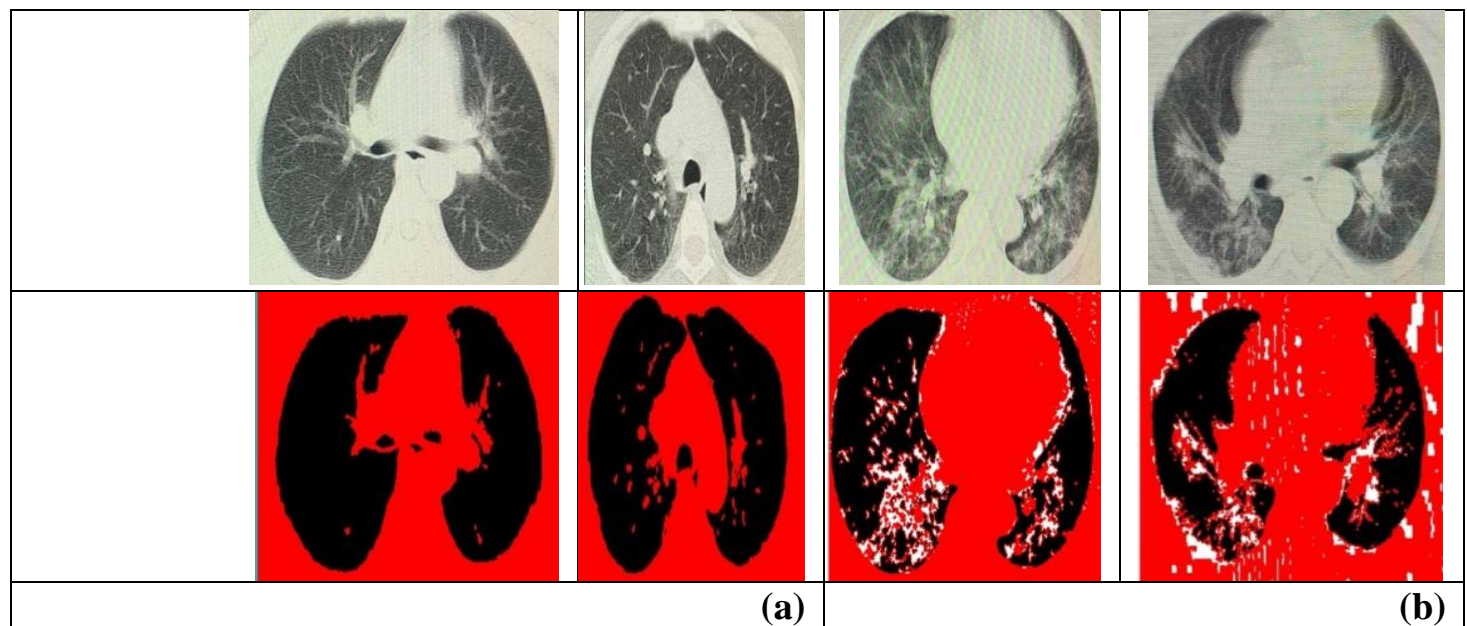

Fig. 1 - Present (a) implementation of Supervisors classification of normal lung images , (b) implementation of Supervisors classification of abnormal lung images

Fig. 1 - show the result of the Supervisors classification of normal lung images are better than the result of the Supervisors classification of abnormal lung images.

B- unSupervisors classification (An iterative method (Isodata) method) are applied on normal and abnormal lung images as shown in Fig. 2

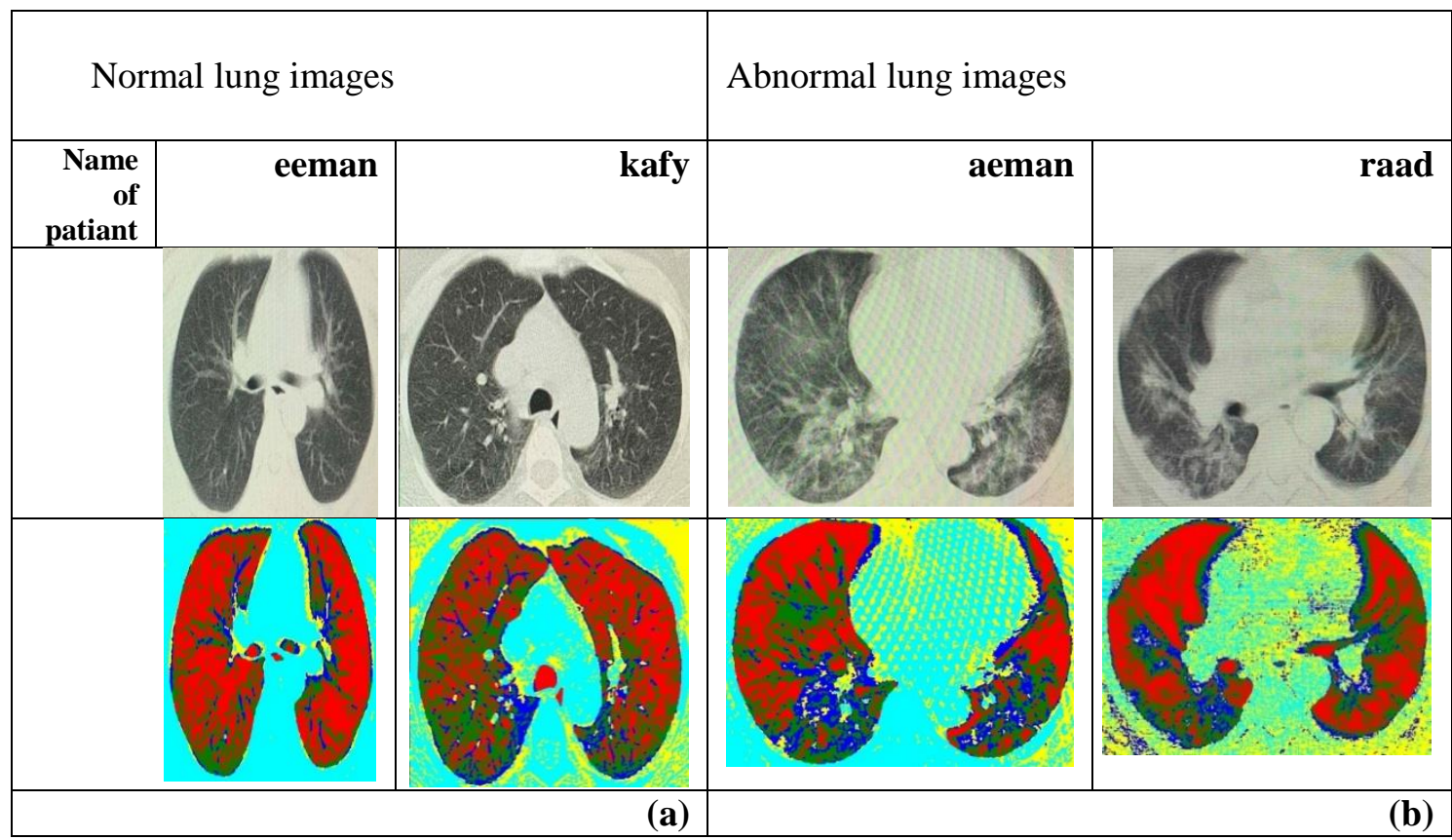

Fig. 2 - Present (a) implementation of unSupervisors classification of normal lung images (b) implementation of unSupervisors classification of abnormal lung images.

Fig. 2 - show the result of unSupervisors classification of abnormal lung images are better than the result of unSupervisors classification of normal lung images.

Fig. 1 - and Fig. 2 - show qualitative (from an appearance of the image) of abnormal lung images after applying Supervisors classification are better than qualitative of abnormal lung images after applying unsupervisors classification to detect the virus with a white color in the lower lobes of the lung 
2. Comparison between Supervised and unsupervised classification quantitative properties

Matlab program has been used to found quantitative properties of supervisor and unsupervisor classification. Quantitative Properties used: kurtosis, skewness, entropy, Stander deviation (STD) mean and histogram.

histogram of Supervisors and UnSupervisors classification lung images show on Fig. 3

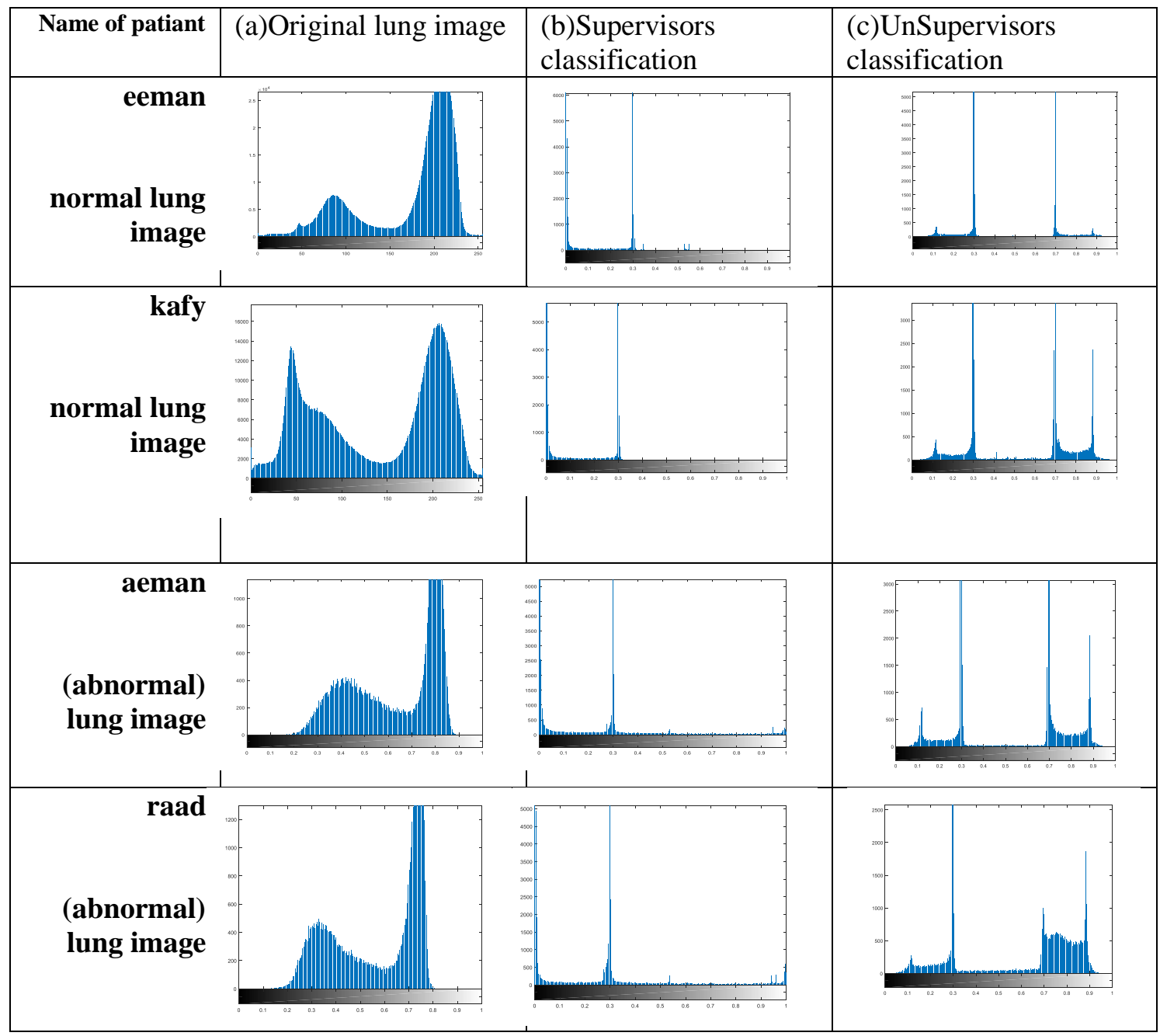

Fig. 3 - Present (a) a histogram of original (normal and abnormal) lung images. (b) a histogram of Supervisors classified (normal and abnormal) lung images (c) a histogram of unsupervisors classified (normal and abnormal) lung images.

Fig. 3 - show a histogram of abnormal lung images after applying Supervisors classification appear a new value in the end of gray level, which means increasing in a white region which it does not appear in the normal lung images, that means there is a coronavirus in lung images. So the histogram shows Supervisors classification are better than Supervisors classification to detect the coronavirus.

\begin{tabular}{|l|l|l|l|l|l|}
\hline & mean & $\begin{array}{c}\text { Stander } \\
\text { deviation(STD) }\end{array}$ & entropy & skewness & kurtosis \\
\hline Eeman(normal) & & \multicolumn{3}{|l|}{} \\
\hline
\end{tabular}




\begin{tabular}{|c|c|c|c|c|c|}
\hline Original lung image & 0.6014 & 0.2352 & 6.6914 & $0.0383-$ & 1.2142 \\
\hline Supervisors classification & 0.1738 & 0.1478 & 2.3003 & $0.2018-$ & 1.3262 \\
\hline $\begin{array}{l}\text { UnSupervisors } \\
\text { classification }\end{array}$ & 0.4848 & 0.2189 & 3.2266 & 0.0850 & 1.3301 \\
\hline \multicolumn{6}{|l|}{ kafy(normal) } \\
\hline Original lung image & 0.5763 & 0.2455 & 7.2018 & $0.0075-$ & 1.2984 \\
\hline Supervisors classification & 0.1597 & 0.1447 & 2.5077 & $0.1273-$ & 1.0494 \\
\hline $\begin{array}{l}\text { UnSupervisors } \\
\text { classification }\end{array}$ & 0.4950 & 0.2443 & 4.8221 & 0.2060 & 1.4317 \\
\hline \multicolumn{6}{|l|}{ aeman(abnormal) } \\
\hline Original lung image & 0.6412 & 0.1853 & 6.8470 & $0.5579-$ & 1.7798 \\
\hline Supervisors classification & 0.2517 & 0.2029 & 3.8982 & 1.2541 & 6.0780 \\
\hline $\begin{array}{l}\text { UnSupervisors } \\
\text { classification }\end{array}$ & 0.5270 & 0.2505 & 5.1217 & $0.1131-$ & 1.4224 \\
\hline \multicolumn{6}{|l|}{$\operatorname{radd}(a b n o r m a l)$} \\
\hline Original lung image & 0.5841 & 0.1810 & 6.5968 & $0.5883-$ & 1.7177 \\
\hline Supervisors classification & 0.2750 & 0.2168 & 4.0351 & 1.3561 & 5.9657 \\
\hline $\begin{array}{l}\text { UnSupervisors } \\
\text { classification }\end{array}$ & 0.5421 & 0.2604 & 5.9739 & $0.0569-$ & 1.3090 \\
\hline
\end{tabular}

Table (1) displays the result value of quantitative Properties for original lung images and classified images

Table (1) Show the value of mean, Stander deviation (STD), entropy of abnormal lung images are higher than value of normal lung images after applying Supervisors and unSupervisors classification. The value of kurtosis and skewness of abnormal lung images are higher than the value of normal lung images after applying Supervisors classification. The value of kurtosis and skewness of normal lung images are higher than the value of abnormal lung images after applying un-Supervisors classification. The value of kurtosis and skewness of abnormal lung images are higher than the value normal lung images of original lung images. Quantitative Properties such as (kurtosis, skewness) of original lung images are similar in rising to value after applying Supervisors classification on it, so Supervisors method is better than un-Supervisors method to distinguishing between normal and abnormal lung images.

\section{Conclusion}

The conclusion can be drawn as follows:

1- Qualitative properties (from an appearance of the image) of abnormal lung images after applying Supervisors classification are better than qualitative of abnormal lung images after applying unsupervisors classification to detect the virus with a white color in the lower lobes of the lung.

2- Quantitative properties (i.e. kurtosis, skewness, entropy, Stander deviation (STD), mean values) had useful distinguishing results between normal and abnormal lung images.

3- The value of mean, Stander deviation (STD), entropy of abnormal lung images are higher than the value of normal lung images after applying Supervisors and unSupervisors classification. The value of kurtosis and skewness of abnormal lung images are higher than the value of normal lung images after applying Supervisors classification. The value of kurtosis and skewness of normal lung images are higher than the value of abnormal lung images after applying unSupervisors classification. The value of kurtosis and skewness of 
abnormal lung images are higher than the value normal lung images of original lung images. Quantitative Properties such as (kurtosis, skewness) of original lung images are similar in rising to value after applying Supervisors classification on it, so Supervisors method is better than unSupervisors method to distinguishing between normal and abnormal lung images.

4- A histogram of abnormal lung images after applying Supervisors classification appear a new value in the end of gray level, which means increasing in a white region which it does not appear in the normal lung images, that means there is a coronavirus in lung images. So the histogram shows Supervisors classification better than Supervisors classification to detect the coronavirus.

Conflict of Interest: The authors declare that they have no conflict of interest.

\section{Acknowledgements:}

We are grateful for Al shaikh zaeid Hospital for cooperating with us to give the image.

\section{Reference}

[1] Anil K. Jain, Fundamentals of Digital Image Processing, Prentice Hall New, Jersey 1989. [2]https://pubs.rsna.org/doi/10.1148/ryai.2020200048.

[3]https://www.newsweek.com/ct-scans-coronavirus-lungs-covid-19-sufferers-1491997.

[4]https://pubs.rsna.org/doi/10.1148/radiol.2020200370.

[5]https://www.shiftcomm.com/insights/understanding-qualitative-quantitative-analysis/.

[6] Gonzalez R.C, Wintz P., Digital Image Processing, second Edition .Addison-Wesley2002. 